\title{
ON GENERATING POINTS OF A LATTICE IN THE REGION
}

$$
\left|x_{1}^{2}+x_{2}^{2}-x_{3}^{2}\right| \leqq 1
$$

by D. M. E. FOSTER

(Received 23 April, 1963)

1. A lattice $\Lambda_{n}$ in $n$-dimensional Euclidean space $E_{n}$ consists of the aggregate of all points with coordinates $\left(x_{1}, \ldots, x_{n}\right)$, where

$$
x_{r}=\sum_{s=1}^{n} \alpha_{r s} u_{s} \quad(r=1, \ldots, n), u_{1}, \ldots, u_{n}=0, \pm 1, \pm 2, \ldots,
$$

for some real $\alpha_{r s}(r, s=1, \ldots, n)$, subject to the condition $\left\|\alpha_{r s}\right\|_{n n} \neq 0$. The determinant $\Delta_{n}$ of $\Lambda_{n}$ is defined by the relation $\Delta_{n}= \pm\left\|\alpha_{r s}\right\|_{n n}$, the sign being chosen to ensure that $\Delta_{n}>0$. If $A_{1}, \ldots, A_{n}$ are the $n$ points of $\Lambda_{n}$ havirig coordinates $\left(\alpha_{11}, \alpha_{21}, \ldots, \alpha_{n 1}\right), \ldots,\left(\alpha_{1 n}, \alpha_{2 n}, \ldots, \alpha_{n n}\right)$, respectively, then every point of $\Lambda_{n}$ may be expressed in the form

$$
u_{1} A_{1}+\ldots+u_{n} A_{n},
$$

and $A_{1}, \ldots, A_{n}$, together with the origin $O$, are said to generate $\Lambda_{n}$. This particular set of generating points is not unique; it may be proved that a necessary and sufficient condition that $n$ points of $\Lambda_{n}$ should generate the lattice is that the $n \times n$ determinant formed by their $x$ coordinates should be $\pm \Delta_{n}$, or, equivalently, that the $n \times n$ determinant formed by their corresponding $u$-coordinates should be \pm 1 .

The problem of finding infinite regions in $E_{n}$ which contain the origin and $n$ further generating points of $\Lambda_{n}$ has already been considered by Minkowski. In particular, Minkowski [13] proved by simple geometrical arguments that the region

$$
\left|x_{1} x_{2}\right| \leqq \frac{1}{2} \Delta_{2}
$$

always contains two generating points of $\Lambda_{2}$. Chalk [3] obtained a generalisation of this result, and later suggested the following conjecture [4] which he proved for $n=3$ and 4 .

CONJECTURE. There exist $n$ lattice points generating $\Lambda_{n}$ in the region

$$
\left|x_{1} x_{2} \ldots x_{n}\right| \leqq \frac{1}{2^{n-1}} \Delta_{n} .
$$

Clearly the conjectured inequality, if true for general $n$, would be best possible when the lattice $\Lambda_{n}$ is of the form

$$
x_{i}=u_{i}+\frac{1}{2} u_{n} \quad(i=1, \ldots, n-1), \quad x_{n}=u_{n} .
$$

Further results of a slightly different nature concerning generating points of $E_{2}$ and $E_{3}$ have also been obtained by Chalk and Rogers [6], Barnes [1] and Oppenheim![15]. 
Our object is to prove the following two theorems, which yield information about sets of generating points of $\Lambda_{3}$ in the three dimensional region

$$
\left|x_{1}^{2}+x_{2}^{2}-x_{3}^{2}\right| \leqq 1
$$

THEOREM 1. If $\Lambda_{3}$ has a point, other than the origin, on the surface $x_{1}^{2}+x_{2}^{2}-x_{3}^{2}=0$, then the region

$$
\left|x_{1}^{2}+x_{2}^{2}-x_{3}^{2}\right| \leqq \Delta_{3}^{2 / 3}
$$

contains a set of generating points of $\Lambda_{3}$.

THEOREM 2. If $\Lambda_{3}$ has no point, other than the origin, on the surface $x_{1}^{2}+x_{2}^{2}-x_{3}^{2}=0$, then the region

$$
\left|x_{1}^{2}+x_{2}^{2}-x_{3}^{2}\right| \leqq\left(\frac{27}{25} \Delta_{3}^{2}\right)^{1 / 3}
$$

contains a set of generating points of $\Lambda_{3}$.

We shall show that the inequalities (1) and (2) are best possible. Before doing so, however, it is convenient to restate Theorems 1 and 2 in terms of indefinite quadratic forms in three variables. For, if $\Lambda_{3}$ is given by equations of the form

$$
x_{r}=\sum_{s=1}^{3} \alpha_{r s} u_{s} \quad(r=1,2,3)
$$

where $\Delta_{3}= \pm\left\|\alpha_{r s}\right\|_{33}$, then clearly $x_{1}^{2}+x_{2}^{2}-x_{3}^{2}$ may be expressed as an indefinite quadratic form

$$
q\left(u_{1}, u_{2}, u_{3}\right)=\sum_{r=1}^{3} \sum_{s=1}^{3} a_{r s} u_{r} u_{s} \quad\left(a_{r s}=a_{s r}\right)
$$

for appropriate $a_{r s}(r, s=1,2,3)$, with determinant $D_{3}=\left\|a_{r s}\right\|_{33}$. On comparison of determinants we see that

$$
D_{3}=-\Delta_{3}^{2}<0
$$

Two quadratic forms $q\left(u_{1}, \ldots, u_{n}\right), Q\left(U_{1}, \ldots, U_{n}\right)$ are said to be equivalent, and we write $q \sim Q$, if $q$ can be transformed into $Q$ by an integral unimodular substitution of the form

$$
u_{r}=\sum_{s=1}^{n} p_{r s} U_{s} \quad(r=1, \ldots, n)
$$

where the $p_{r s}$ are integers with determinant $\left\|p_{r s}\right\|= \pm 1$. The following Theorems $1^{*}$ and $2^{*}$, which are expressed in terms of quadratic forms, contain the assertions of Theorems 1 and 2 , respectively, and we prove them in this form. 
THEOREM 1*. If $q\left(u_{1}, u_{2}, u_{3}\right)$ represents zero non-trivially, then it is equivalent to a form for which

$$
\left|a_{i i}\right| \leqq\left|D_{3}\right|^{1 / 3} \quad(i=1,2,3),
$$

with strict inequality unless $q \sim \lambda q_{0}$ or $\lambda q_{1}$, where

and

$$
\begin{aligned}
& q_{0}\left(u_{1}, u_{2}, u_{3}\right)=2 u_{1} u_{2}+u_{3}^{2} \\
& q_{1}\left(u_{1}, u_{2}, u_{3}\right)=2 u_{1} u_{2}+u_{2}^{2}+u_{2} u_{3}+u_{3}^{2} .
\end{aligned}
$$

THEOREM 2*. If $q\left(u_{1}, u_{2}, u_{3}\right)$ does not represent zero non-trivially, then it is equivalent to a form for which

$$
\left|a_{i i}\right| \leqq\left(\frac{27}{25}\left|D_{3}\right|\right)^{1 / 3} \quad(i=1,2,3)
$$

with strict inequality unless $q \sim \lambda q_{2}$, where

$$
q_{2}\left(u_{1}, u_{2}, u_{3}\right)=u_{1}^{2}+u_{1} u_{2}-u_{2}^{2}+\frac{5}{2} u_{3}^{2} .
$$

In a recent paper [5], Dr J. H. H. Chalk has obtained a striking result for a certain class of quadratic forms in an even number of variables. He has shown that if

$$
q\left(u_{1}, \ldots, u_{2 m}\right)=\sum_{r=1}^{2 m} \sum_{s=1}^{2 m} a_{r s} u_{r} u_{s} \quad\left(a_{r s}=a_{s r}\right)
$$

is an indefinite form in $u_{1}, \ldots, u_{2 m}$ of signature zero and determinant $D_{2 m}=\left\|a_{r s}\right\|_{2 m, 2 m} \neq 0$, then it is equivalent to a form for which

with equality when

$$
\left|a_{l i}\right| \leqq\left|D_{2 m}\right|^{1 / 2 m} \quad(i=1, \ldots, 2 m)
$$

$$
q\left(u_{1}, \ldots, u_{2 m}\right)=\sum_{r=1}^{m-1}\left(u_{2 r-1}^{2}-u_{2 r}^{2}\right)+2 u_{2 m-1} u_{2 m} .
$$

The proof of Theorem $1^{*}$ in $\$ 2$ divides into two cases, in one of which we use an elementary result in the theory of continued fractions (Lemma 1 ) to replace the inequalities (1)* by

$$
\left|a_{i i}\right|<\varepsilon \quad(i=1,2,3)
$$

for any $\varepsilon>0$. The other case is less trivial and the proof depends upon Lemma 2, which gives a useful inequality for a quadratic in a single integral variable. The lemma is not new and is a corollary of Lemma 5 of Davenport [7], but a proof is given for convenience. The use of this lemma could be avoided by a direct appeal to a theorem of Macbeath [11] on a quadratic polynomial in two variables.

The proof of Theorem $2^{*}$ is rather different and is based upon Lemma 2 and three further lemmas. Lemma 3, which is needed as a starting point for the proof of the theorem, is classical and gives the first " minimum " for an indefinite quadratic form in three variables. Lemma 4 
is a straightforward extension, to a two-dimensional asymmetric hyperbolic region, of Minkowski's original theorem on generating points of $\Lambda_{2}$. The result stated in Lemma 5 is a special case of a recent theorem of Watson on values of a non-zero binary quadratic form.

I am very grateful to $\mathrm{Dr}$ J. H. H. Chalk for suggesting this problem to me and for his valuable help and advice during my work on it. I should also like to thank $\mathrm{Dr} G$. L. Watson for his helpful suggestions in improving the presentation.

2. For the proof of Theorem $1^{*}$ we require the following two lemmas.

LemMa 1. If $\alpha$ is a given positive irrational number and $\varepsilon>0$, then the inequalities

$$
0<\left|q_{n} \alpha-p_{n}\right|<\varepsilon \text { and } 0<\left|q_{n+1} \alpha-p_{n+1}\right|<\varepsilon
$$

are always soluble in integer pairs $\left(p_{n}, q_{n}\right)$ and $\left(p_{n+1}, q_{n+1}\right)$ with $p_{n} q_{n+1}-p_{n+1} q_{n}=1$.

Proof. Take $p_{n} / q_{n}$ and $p_{n+1} / q_{n+1}$ to be successive convergents to the continued fraction for $\alpha$ with $n$ odd and sufficiently large.

LEMMA 2. If $a, \alpha, t$ are any constants satisfying

$$
\begin{aligned}
& 0<a<1, \\
& 0 \leqq t^{2}<1+\frac{1}{4} a^{2},
\end{aligned}
$$

then the inequalities

$$
\left|a(u+\alpha)^{2}-a^{-1} t^{2}\right|<1
$$

are always soluble for an integer $u$.

Proof. We write

$$
f(u)=a(u+\alpha)^{2}-a^{-1} t^{2}
$$

for convenience. If $t^{2}<a$, we choose an integer $u$ satisfying

$$
|u+\alpha|<a^{-1}\left(t^{2}+a\right)^{1 / 2},
$$

which is possible since

$$
a^{-1}\left(t^{2}+a\right)^{1 / 2}>a^{-1 / 2}>1,
$$

by (3) and (4). With this value of $u$ we have

$$
-1<-a^{-1} t^{2}<f(u)<1 .
$$

If $t^{2} \geqq a$, let $u$ denote the integer for which

$$
a^{-1}\left(t^{2}+a\right)^{1 / 2}-1 \leqq u+\alpha<a^{-1}\left(t^{2}+a\right)^{1 / 2} .
$$


We have, successively,

$$
\begin{aligned}
t^{2} & <1+\frac{1}{t} a^{2}, \\
4\left(t^{4}-a^{2}\right) & <4 t^{4}-4 t^{2} a^{2}+a^{4}, \\
2\left(t^{4}-a^{2}\right)^{1 / 2} & <2 t^{2}-a^{2}, \\
a^{2} & <2 t^{2}-2\left(t^{4}-a^{2}\right)^{1 / 2}, \\
1 & <a^{-1}\left(t^{2}+a\right)^{1 / 2}-a^{-1}\left(t^{2}-a\right)^{1 / 2} .
\end{aligned}
$$

By (6) and (7) we see that $u$ satisfies

$$
a^{-1}\left(t^{2}-a\right)^{1 / 2}<u+\alpha<a^{-1}\left(t^{2}+a\right)^{1 / 2},
$$

and (5) now follows.

Proof of Theorem $1^{*}$. By considering a positive multiple of $q=q\left(u_{1}, u_{2}, u_{3}\right)$ in place of $q$, if necessary, we may assume that $\left|D_{3}\right|=1$. Then it suffices to prove that, unless $q \sim \lambda q_{0}$ or $2 q_{1}$, the inequalities

$$
\left|q\left(u_{1 s}, u_{2 s}, u_{3 s}\right)\right|<1 \quad(s=1,2,3)
$$

are soluble in integers $\left(u_{1 s}, u_{2 s}, u_{3 s}\right)$, with $\left\|u_{r s}\right\|_{33}=1$, since the integral unimodular substitution

$$
u_{r}=\sum_{s=1}^{3} u_{r s} U_{s} \quad(r=1,2,3)
$$

will transform $q$ into a form each of whose diagonal coefficients is less than 1 in absolute value.

As $q$ represents zero non-trivially, we may suppose, after applying an integral unimodular substitution to the variables, that $a_{11}=0$, and $q$ now takes the form

$$
q\left(u_{1}, u_{2}, u_{3}\right)=2\left(a_{12} u_{2}+a_{13} u_{3}\right) u_{1}+a_{22} u_{2}^{2}+2 a_{23} u_{2} u_{3}+a_{33} u_{3}^{2} \text {. }
$$

Since $\left|D_{3}\right| \neq 0$, the coefficients $a_{12}, a_{13}$ cannot both be zero. By interchanging $u_{2}, u_{3}$, if necessary, we may suppose that $a_{12} \neq 0$. Two cases now arise, according as the ratio $a_{13} / a_{12}$ is irrational or rational.

Suppose first that $a_{13} / a_{12}$ is irrational and let $\varepsilon>0$. By changing the signs of $u_{2}, u_{3}$, if necessary, we may assume that $a_{12}>0, a_{13}<0$.

Choose $\left(u_{11}, u_{21}, u_{31}\right)=(1,0,0)$. By Lemma 1 , since $\left(\varepsilon / a_{12}\right)>0$, there exist integer pairs $\left(u_{22}, u_{32}\right)$ and $\left(u_{23}, u_{33}\right)$, with $u_{22} u_{33}-u_{23} u_{32}=1$, satisfying

$$
0<\left|u_{2 s}+\frac{a_{13}}{a_{12}} u_{3 s}\right|<\frac{\varepsilon}{a_{12}} \quad(s=2,3) \text {. }
$$

For each pair $\left(u_{2 s}, u_{3 s}\right)(s=2,3)$, we can always choose a corresponding integer $u_{1}=u_{1}$ s $(s=2,3)$ satisfying

$$
\left|q\left(u_{1 s}, u_{2 s}, u_{3 s}\right)\right| \leqq \varepsilon
$$


and (8) follows with the triads $(1,0,0),\left(u_{12}, u_{22}, u_{32}\right)$ and $\left(u_{13}, u_{23}, u_{33}\right)$, since $\varepsilon$ may be arbitrarily small.

Now suppose that $a_{13} / a_{12}=q / p$ where $p, q$ are integers with $(p, q)=1$ and $q \neq 0$ (i.e. $a_{13} \neq 0$ ). It is known that there exist integers $p^{\prime}, q^{\prime}$, with $\left(p^{\prime}, q^{\prime}\right)=1$, satisfying $p q^{\prime}-p^{\prime} q=1$. Then the integral unimodular substitution given by

$$
u_{1}^{\prime}=u_{1}, \quad u_{2}^{\prime}=p u_{2}+q u_{3}, \quad u_{3}^{\prime}=p^{\prime} u_{2}+q^{\prime} u_{3}
$$

will reduce $q$ to the form

$$
q\left(u_{1}, u_{2}, u_{3}\right)=2 b_{12} u_{1} u_{2}+b_{22} u_{2}^{2}+2 b_{23} u_{2} u_{3}+b_{33} u_{3}^{2},
$$

for appropriate $b_{12}, \ldots, b_{33}$. If $a_{13}=0$, the above substitution is not required. Comparing determinants we see that

$$
b_{12}^{2}\left|b_{33}\right|=\left|D_{3}\right|=1 \text {. }
$$

- If $\left|b_{12}\right|<1$, the result is easily proved, by choosing the triads $(1,0,0),\left(u_{12}, 1,0\right)$ and $\left(u_{13}, 1,1\right)$, where $u_{12}, u_{13}$ are the integers satisfying

and

$$
\begin{gathered}
\left|2 b_{12} u_{12}+b_{22}\right| \leqq\left|b_{12}\right|<1 \\
\left|2 b_{12} u_{13}+b_{22}+2 b_{23}+b_{33}\right| \leqq\left|b_{12}\right|<1 .
\end{gathered}
$$

Now suppose that $\left|b_{12}\right|>1$ and hence $\left|b_{33}\right|<1$, by (9). We first choose the triads $(1,0,0)$ and $(0,0,-1)$. Then taking $u_{2}=u_{23}=1$, we have, on re-arranging,

$$
q\left(u_{1}, 1, u_{3}\right)=b_{33}\left(u_{3}+\frac{b_{23}}{b_{33}}\right)^{2}+2 b_{12} u_{1}+b_{22}-\frac{b_{23}^{2}}{b_{33}} .
$$

By considering $-q\left(u_{1}, 1, u_{3}\right)$, if necessary, we may suppose that

$$
0<b_{33}<1 \text {. }
$$

Let $u_{1}=u_{13}$ be the integer satisfying

If

$$
\begin{gathered}
1-\frac{1}{4} b_{33}-2 b_{12} \leqq 2 b_{12} u_{13}+b_{22}-\frac{b_{23}^{2}}{b_{33}}<1-\frac{1}{4} b_{33} . \\
0 \leqq 2 b_{12} u_{13}+b_{22}-\frac{b_{23}^{2}}{b_{33}}<1-\frac{1}{4} b_{33},
\end{gathered}
$$

we choose an integer $u_{3}=u_{33}$ satisfying

$$
\left|u_{33}+\frac{b_{23}}{b_{33}}\right| \leqq \frac{1}{2}
$$

and then (8) follows. Thus we are left to consider the case in which

$$
q\left(u_{13}, 1, u_{3}\right)=b_{33}\left(u_{3}+\frac{b_{23}}{b_{33}}\right)^{2}-\lambda
$$


where

$$
0<b_{33} \lambda \leqq 4 b_{33}^{2}-b_{33}+2 b_{33}^{1 / 2},
$$

and hence, since $0<b_{33}<1$, we have

$$
0<b_{33} \lambda<\frac{1}{4} b_{33}^{2}+1 .
$$

By Lemma 2, with $a=b_{33}, \alpha=b_{23} / b_{33}, t^{2}=b_{33} \lambda$, it follows that there is an integer $u_{3}=u_{33}$ satisfying

$$
\left|q\left(u_{13}, 1, u_{33}\right)\right|<1 .
$$

It remains to consider the case in which $\left|b_{12}\right|=1,\left|b_{33}\right|=1$. By changing, if necessary, the sign of $q$ or the sign of $u_{1}$ or both we may suppose that

$$
q\left(u_{1}, u_{2}, u_{3}\right)=2 u_{1} u_{2}+b_{22} u_{2}^{2}+2 b_{23} u_{2} u_{3}+u_{3}^{2} .
$$

Further, by absorbing integral multiples of $u_{2}, u_{3}$ into $u_{1}$ and changing the sign of $u_{3}$, if necessary, we may suppose that

$$
\left|b_{22}\right| \leqq 1 \text { and } 0 \leqq 2 b_{23} \leqq 1 .
$$

If $\left|b_{22}\right|<1$, the congruences

$$
b_{22} \pm 2 b_{23} \equiv 0(\bmod 2)
$$

together imply that $b_{22}=b_{23}=0$. Thus if $u_{13}, u_{13}^{\prime}$ are integers satisfying

and

$$
\left|2 u_{13}+b_{22}+2 b_{23}+1\right| \leqq 1
$$

$$
\left|2 u_{13}^{\prime}-b_{22}+2 b_{23}-1\right| \leqq 1 \text {, }
$$

respectively, then it follows that $|q|<1$ for the triads $(1,0,0),(0,1,0)$ and $\left(u_{13}, 1,1\right)$ or $\left(u_{13}^{\prime},-1,1\right)$, unless

$$
q=q_{0}=2 u_{1} u_{2}+u_{3}^{2} .
$$

If $\left|b_{22}\right|=1$, then $q$ is equivalent to the form

$$
q\left(u_{1}, u_{2}, u_{3}\right)=2 u_{1} u_{2}+u_{2}^{2}+2 b_{23} u_{2} u_{3}+u_{3}^{2} .
$$

Let $u_{13}$ be an integer satisfying

$$
\left|u_{13}+b_{23}-1\right| \leqq \frac{1}{2} .
$$

Then $|q|<1$ for the triads $(1,0,0),(-1,2,-1)$ and $\left(u_{13},-1,1\right)$, unless $2 b_{23}=1$, in which case

$$
q=q_{1}=2 u_{1} u_{2}+u_{2}^{2}+u_{2} u_{3}+u_{3}^{2} .
$$


3. In this section we prove Theorem $2^{*}$. The proof is independent of Theorem $1^{*}$ and use is made of the following three lemmas.

LEMMA 3. The inequalities

$$
\left|q\left(u_{1}, u_{2}, u_{3}\right)\right| \leqq\left(\frac{2}{3}\left|D_{3}\right|\right)^{1 / 3}
$$

are always soluble in integers $\left(u_{1}, u_{2}, u_{3}\right) \neq(0,0,0)$.

For a proof of this classical result, which is the first of a sequence of minima of an indefinite quadratic form in three variables, see [10]. We observe, in passing, that the particular form relating to the fourth minimum arises as the critical form $q_{2}\left(u_{1}, u_{2}, u_{3}\right)$ in Theorem $2^{*}$.

LEMMA 4. For any $\Gamma>0$, the region

$$
-\Gamma \Delta_{2} \leqq x_{1} x_{2} \leqq \frac{1}{4 \Gamma} \Delta_{2}
$$

always contains two generating points of $\Lambda_{2}$.

Proof. Consider the tangent parallelogram $\Pi_{t}$ defined by

$$
\left|t^{-1} x_{1}+t x_{2}\right| \leqq \sqrt{ }\left(\Delta_{2} / \Gamma\right), \quad\left|t^{-1} x_{1}-t x_{2}\right| \leqq 2 \sqrt{ }\left(\Gamma \Delta_{2}\right)
$$

Clearly $\Pi_{t}$ is symmetrical about the origin, and since it may be transformed by a linear substitution of determinant 2 into a rectangle having area $8 \Delta_{2}$, its area is $4 \Delta_{2}$. By Minkowski's fundamental theorem, $\Pi_{t}$ contains a point of $\Lambda_{2}$ other than the origin $O$. Further, by varying $t$ continuously, we can obtain a parallelogram $\Pi_{t^{\prime}}$, which contains two independent points $P, Q$, say, of $\Lambda_{2}$, other than $O$. Let $P^{\prime}, Q^{\prime}$ be the reflections of $P, Q$ respectively in $O$. If the parallelogram $P Q P^{\prime} Q^{\prime}$ contains points of $\Lambda_{2}$ other than $O$, we simply replace it by a smaller parallelogram. Thus we assume that $P Q P^{\prime} Q^{\prime}$ does not contain any point of $\Lambda_{2}$ other than $O$.

Since $P, Q$ are lattice points, it follows that the area of the parallelogram with sides $O P$, $O Q$ is an integral multiple of $\Delta_{2}$, say $m \Delta_{2}$. The area of the parallelogram $P Q P^{\prime} Q^{\prime}$ is $2 m \Delta_{2}$, and $2 m \Delta_{2} \leqq 4 \Delta_{2}$; consequently two possibilities arise according as $m=1$ or 2 . If $m=1$, the parallelogram with sides $O P, O Q$ has area $\Delta_{2}$, and hence $P, Q$, together with $O$, generate $\Lambda_{2}$. If $m=2$, the parallelogram $P Q P^{\prime} Q^{\prime}$ coincides with the original tangent parallelogram $\Pi_{t}$, and $Q$ and $\frac{1}{2}(P+Q)$, together with $O$, generate $\Lambda_{2}$.

We observe that the two generating points obtained lie entirely inside the region considered if there is no point of $\Lambda_{2}$ on either bounding hyperbola. However, if there is a point of $\Lambda_{2}$ on one of these hyperbolae, the tangent parallelogram $\Pi_{t}$, for suitable $t$, through that point will have on its boundary two basis points lying inside the region, unless there is a primitive point of $\Lambda_{2}$ on the other hyperbola. In this case $\Lambda_{2}$ is of the form

$$
\begin{gathered}
x_{1}=\frac{t}{2} \sqrt{\left(\frac{\Delta_{2}}{\Gamma}\right) u_{1}-t \sqrt{ }\left(\Gamma \Delta_{2}\right) u_{2},} \\
x_{2}=\frac{1}{2 t} \sqrt{\left(\frac{\Delta_{2}}{\Gamma}\right) u_{1}+t^{-1} \sqrt{ }\left(\Gamma \Delta_{2}\right) u_{2} .}
\end{gathered}
$$


Restating the result with $\mu=1 /(2 \Gamma)$, we obtain the following corollary.

COROLLARY. If $\mu>0$ and if $q\left(u_{1}, u_{2}\right)=\left(\alpha u_{1}+\beta u_{2}\right)\left(\gamma u_{1}+\delta u_{2}\right)$ is an indefinite quadratic form in $u_{1}, u_{2}$ of determinant $d=-\frac{1}{4}(\alpha \delta-\beta \gamma)^{2}$, then the inequalities

$$
-\frac{1}{\mu}|d|^{1 / 2}<q\left(u_{1 s}, u_{2 s}\right)<\mu|d|^{1 / 2} \quad(s=1,2)
$$

are always soluble in integers $\left(u_{1 s}, u_{2 s}\right)(s=1,2)$ with $\left\|u_{r s}\right\|=1$, unless

$$
q\left(u_{1}, u_{2}\right) \sim \sqrt{ }(|d|)\left(\mu u_{1}^{2}-\mu^{-1} u_{2}^{2}\right) \text {. }
$$

A proof of the next lemma, due to Watson, is given for convenience as his has not been published. Let

$$
q=q\left(u_{1}, u_{2}\right)=a u_{1}^{2}+2 b u_{1} u_{2}+c u_{2}^{2}
$$

denote an indefinite quadratic form in $u_{1}, u_{2}$ which does not represent zero non-trivially and has determinant

$$
d=a c-b^{2}<0 .
$$

Denote by $P, N$ the lower bounds of the positive values of $q,-q$, respectively, for all integers $\left(u_{1}, u_{2}\right) \neq(0,0)$.

\section{LEMMA 5.}

with equality when

$$
P N \leqq \frac{4}{5}|d|
$$

$$
q\left(u_{1}, u_{2}\right)=\lambda\left(u_{1}^{2}+u_{1} u_{2}-u_{2}^{2}\right)
$$

Proof. $\dagger$ We suppose $P N \neq 0$, for otherwise the result is obvious. Also if $P=N$ the result is well known [12], since

$$
P=N \leqq \sqrt{ }\left(\frac{4}{5}|d|\right)
$$

By changing the sign of $q$, if necessary, we may suppose that

$$
N<P \text {. }
$$

Hence

$$
N \leqq \sqrt{ }\left(\frac{4}{5}|d|\right)
$$

If we consider a suitable multiple of $q$ instead of $q$, we may take $P=1$, and it now suffices to prove that

$$
N \leqq \frac{4}{5}|d|
$$

Let $\varepsilon>0$. After applying an appropriate unimodular substitution to the variables $u_{1}, u_{2}$, we may assume that

$$
1 \leqq a<1+\varepsilon, \quad \frac{1}{2} a \leqq b \leqq a .
$$

$\uparrow$ The proof given here is an adaptation of that of Dr Watson, who has very kindly let me reproduce it. เ. 
By our hypothesis concerning $P$ and $N$, it follows that either $q \leqq-N$ or $q \geqq 1$ for all integers $\left(u_{1}, u_{2}\right) \neq(0,0)$.

The inequality (12) follows easily if $|d| \geqq \frac{5}{4}$. For in this case we have

$$
N \leqq \sqrt{ }\left(\frac{4}{5}|d|\right) \leqq \frac{4}{5}|d|,
$$

by (11). Thus suppose now that

$$
|d|<\frac{5}{4} .
$$

Since

$$
a c-b^{2}=d<0
$$

we have

$$
a c<b^{2} \leqq a^{2},
$$

by (13), and hence

$$
c<a .
$$

Thus either (i) $1 \leqq c<1+\varepsilon$, or (ii) $c<0$.

In the first case

$$
q(-1,1)=a-2 b+c,
$$

and by (13), (15) and the choice of $c$, we have

$$
1-2(1+\varepsilon)+1<q(-1,1)<1+\varepsilon-2+1+\varepsilon,
$$

since, using (13), $b \geqq \frac{1}{2} a>0$ and $(1+\varepsilon)^{2}>b^{2}>a c \geqq 1$. Thus

$$
-2 \varepsilon<q(-1,1)<2 \varepsilon,
$$

which is impossible if $\varepsilon$ is sufficiently small. Hence only the second case can arise, and we have therefore

$$
c \leqq-N .
$$

Now

$$
\begin{aligned}
|d|=a|c|+b^{2} & \geqq a N+\frac{1}{4} a^{2}, \quad \text { by }(16), \\
& \geqq N+\frac{1}{4}, \quad \text { by }(13),
\end{aligned}
$$

so that

$$
\begin{aligned}
N & \leqq|d|-\frac{1}{4} \\
& <\frac{4}{5}|d|, \quad \text { by (14). }
\end{aligned}
$$

Proof of Theorem 2*. By considering a suitable positive multiple of $q=q\left(u_{1}, u_{2}, u_{3}\right)$ in place of $q$, if necessary, we can take $D_{3}=-25 / 27$. Then, as in the proof of Theorem $1^{*}$, it suffices to prove that, unless $q \sim \lambda q_{2}$, the inequalities

$$
\left|q\left(u_{1 s}, u_{2 s}, u_{3 s}\right)\right|<1 \quad(s=1,2,3)
$$

are always soluble in integers $\left(u_{1 s}, u_{2 s}, u_{3 s}\right)(s=1,2,3)$, with $\left\|u_{r s}\right\|_{33}=1$. 
If $M$ denotes the lower bound of $\left|q\left(u_{1}, u_{2}, u_{3}\right)\right|$ over all integer triads $\left(u_{1}, u_{2}, u_{3}\right) \neq$ $(0,0,0)$, then, by a weaker form of Lemma 3 , we have

$$
0 \leqq M<9 / 10 .
$$

Suppose first that $M=0$. Then, for any $\varepsilon>0$, the inequalities

$$
0<\left|q\left(u_{1}, u_{2}, u_{3}\right)\right|<\varepsilon
$$

are always soluble in integers $u_{1}, u_{2}, u_{3}$, and it follows that the inequalities

$$
0<q\left(u_{1}, u_{2}, u_{3}\right)<\varepsilon
$$

are also soluble for any $\varepsilon>0$, by a theorem of Oppenheim [14].

Now suppose that $M \neq 0$, and choose $\varepsilon$ so that

$$
0 \leqq \varepsilon<1 / 81 \text {. }
$$

By the definition of $M$, there are coprime integers $u_{1}, u_{2}, u_{3}$ satisfying

$$
0<M \leqq|q|<M /(1-\varepsilon)<1 .
$$

Thus, if the inequalities $0<q<1$ are insoluble in integers $u_{1}, u_{2}, u_{3}$, then the inequalities $0<-q<1$ are soluble in integers $u_{1}, u_{2}, u_{3}$.

In either case, therefore, after applying a suitable unimodular substitution to the variables $u_{1}, u_{2}, u_{3}$, we may ensure that either

or

$$
\begin{aligned}
& \text { (i) } 0<a_{11}<1 \\
& \text { (ii) } 0<-a_{11}<M /(1-\varepsilon)<1 \text {, }
\end{aligned}
$$

and in case (ii) the inequalities $0<q<1$ are insoluble in integers $u_{1}, u_{2}, u_{3}$.

Case (i). We may write

$$
q\left(u_{1}, u_{2}, u_{3}\right)=a_{11}\left(u_{1}+c_{2} u_{2}+c_{3} u_{3}\right)^{2}+q_{1}\left(u_{2}, u_{3}\right),
$$

for suitable constants $c_{2}, c_{3}$ and $q_{1}\left(u_{2}, u_{3}\right)$, which is an indefinite quadratic form in $u_{2}, u_{3}$ of determinant $-25 /\left(27 a_{11}\right)$. By the corollary to Lemma 4 , with $\mu=\left\{\left(4-a_{11}\right)\left(27 a_{11}\right)^{\frac{t}{3}}\right\} / 20$, there exist integer pairs $\left(u_{22}, u_{32}\right)$ and $\left(u_{23}, u_{33}\right)$, with $u_{22} u_{33}-u_{23} u_{32}=1$, satisfying

$$
-\frac{100}{\left(4-a_{11}\right) 27 a_{11}}<q_{1}\left(u_{2 s}, u_{3 s}\right)<\frac{4-a_{11}}{4} \quad(s=2,3) \text {, }
$$

unless

$$
q_{1}\left(u_{2}, u_{3}\right) \sim\left(\mu u_{2}^{2}-\mu^{-1} u_{3}^{2}\right)\left\{25 /\left(27 a_{11}\right)\right\}^{\frac{1}{2}}
$$

If

$$
0 \leqq q_{1}\left(u_{2 s}, u_{3 s}\right)<\frac{4-a_{11}}{4}
$$


for some $s=2,3$, we choose an integer $u_{1 s}$ satisfying

and then

$$
\left|u_{1 s}+c_{2} u_{2 s}+c_{3} u_{3 s}\right| \leqq \frac{1}{2},
$$

$$
\left|q\left(u_{1 s}, u_{2 s}, u_{3 s}\right)\right|<\frac{1}{4} a_{11}+\frac{1}{4}\left(4-a_{11}\right)=1 .
$$

Now suppose that $q_{1}\left(u_{2 s}, u_{3 s}\right)=-\lambda$ for some $s=2,3$, where

$$
0<\lambda<100 /\left\{27 a_{11}\left(4-a_{11}\right)\right\} \text {, by (18), }
$$

i.e.

$$
0<a_{11} \lambda<100 /\left\{27\left(4-a_{11}\right)\right\} \text {. }
$$

In this case we have

$$
q\left(u_{1}, u_{2 s}, u_{3 s}\right)=a_{11}\left(u_{1}+c_{2} u_{2 s}+c_{3} u_{3 s}\right)^{2}-a_{11}^{-1}\left(a_{11} \lambda\right) .
$$

Since $0<a_{11}<1$, we have, successively,

$$
\begin{aligned}
\left(3 a_{11}-2\right)^{2}\left(3 a_{11}-8\right) & \leqq 0, \\
27 a_{11}^{3}-108 a_{11}^{2}+108 a_{11}-32 & \leqq 0 \\
400-27\left(4-a_{11}\right)\left(4+a_{11}^{2}\right) & \leqq 0 \\
100 /\left\{27\left(4-a_{11}\right)\right\} & \leqq\left(4+a_{11}^{2}\right) / 4 .
\end{aligned}
$$

By Lemma 2, with $a=a_{11}, t^{2}=a_{11} \lambda$, there is an integer $u_{1 s}$ satisfying

$$
\left|q\left(u_{1 s}, u_{2 s}, u_{3 s}\right)\right|<1 .
$$

Thus the inequalities (17) follow, with the triads $(1,0,0),\left(u_{12}, u_{22}, u_{32}\right)$ and $\left(u_{13}, u_{23}, u_{33}\right)$.

It remains to consider the case in which

$$
q_{1}\left(u_{2}, u_{3}\right)=\left(\mu u_{2}^{2}-\mu^{-1} u_{3}^{2}\right)\left\{25 /\left(27 a_{11}\right)\right\}^{\frac{1}{2}},
$$

where $\mu=\left\{\left(4-a_{11}\right)\left(27 a_{11}\right)^{ \pm}\right\} / 20$. If we choose $\left(u_{22}, u_{32}\right)=(0,-1)$ and $\left(u_{23}, u_{33}\right)=(1,1)$, then

$$
-\frac{100}{\left(4-a_{11}\right) 27 a_{11}} \leqq q_{1}\left(u_{2 s}, u_{3 s}\right)<\frac{4-a_{11}}{4} \quad(s=2,3),
$$

and (17) again follows, with the triads $(1,0,0),\left(u_{12}, 0,-1\right)$ and $\left(u_{13}, 1,1\right)$, unless $a_{11}=2 / 3$. In this case $\mu=1 / \sqrt{ } 2$, and $q$ is equivalent to

$$
q\left(u_{1}, u_{2}, u_{3}\right)=\frac{2}{3}\left(u_{1}+c_{2}^{\prime} u_{2}+c_{3}^{\prime} u_{3}\right)^{2}+\frac{5}{6}\left(u_{2}^{2}-2 u_{3}^{2}\right)
$$

for some constants $c_{2}^{\prime}, c_{3}^{\prime}$. By absorbing integral multiples of $u_{2}, u_{3}$ into $u_{1}$ and changing the sign of $u_{2}$, if necessary, we may assume that

$$
0 \leqq c_{2}^{\prime} \leqq \frac{1}{2} \text { and } 0 \leqq c_{3}^{\prime}<1 .
$$

We shall show that there are three triads of determinant 1 for which $|q|<1$, unless $c_{2}^{\prime}=\frac{1}{2}$ and $c_{3}^{\prime}=0$. 
If $c_{2}^{\prime} \neq \frac{1}{2}$ and $c_{3}^{\prime} \neq 0$, we choose the triads $(1,0,0),(0,1,0)$ and $(1,0,1) ;$ if $c_{2}^{\prime}=\frac{1}{2}$ and $c_{3}^{\prime} \neq 0$, we choose the triads $(1,0,0),(1,1,-1)$ and $(1,0,1)$; finally, if $c_{2}^{\prime} \neq \frac{1}{2}, c_{3}^{\prime}=0$, we choose the triads $(1,0,0),(1,1,-1)$ and $(0,1,0)$.

In the remaining case, when $\left(c_{2}^{\prime}, c_{3}^{\prime}\right)=\left(\frac{1}{2}, 0\right)$, the unimodular substitution

$$
u_{1}=U_{1}+U_{3}, \quad u_{2}=U_{2}-2 U_{3}, \quad u_{3}=U_{2}-U_{3}
$$

will transform $q$ into the equivalent form $Q=Q\left(U_{1}, U_{2}, U_{3}\right)$, where

$$
\frac{3}{2} Q\left(U_{1}, U_{2}, U_{3}\right)=U_{1}^{2}+U_{1} U_{2}-U_{2}^{2}+\frac{5}{2} U_{3}^{2} \text {. }
$$

It may be verified that $\frac{3}{2} Q$ does not represent zero, and that it has absolute minimum 1 , attained only when $U_{2} \equiv 0(\bmod 2)$.

Before going on to the alternative case, we observe that, if $M=0$, we can ensure that $0<a_{11}<\varepsilon$, and a slight modification of the foregoing proof will yield a result of the type

$$
\left|q\left(u_{1 s}, u_{2 s}, u_{3 s}\right)\right|<\varepsilon \quad(s=1,2,3)
$$

with $\left\|u_{r s}\right\|_{33}=1$.

Case (ii). In this case we write

$$
q\left(u_{1}, u_{2}, u_{3}\right)=-\left|a_{11}\right|\left(u_{1}+d_{2} u_{2}+d_{3} u_{3}\right)^{2}+q_{2}\left(u_{2}, u_{3}\right),
$$

for suitable constants $d_{2}, d_{3}$ and $q_{2}\left(u_{2}, u_{3}\right)$, which is a positive definite quadratic form in $u_{2}, u_{3}$ of determinant $25 /\left(27\left|a_{11}\right|\right)$, and

$$
0<\left|a_{11}\right|<1 \text {. }
$$

After applying an integral unimodular substitution to the variables $u_{2}, u_{3}$, it is known $[8$, Theorem 51] that we can ensure that

$$
q_{2}\left(u_{2}, u_{3}\right)=A u_{2}^{2}+2 B u_{2} u_{3}+C u_{3}^{2}
$$

where

$A C-B^{2}=\frac{25}{27\left|a_{11}\right|},|2 B| \leqq A$ and $0<A \leqq \min \left\{C, \sqrt{\frac{4}{3} \cdot \frac{25}{27\left|a_{11}\right|}}=\sqrt{\frac{100}{81\left|a_{11}\right|}}\right\}$.

We again choose $\left(u_{11}, u_{21}, u_{31}\right)=(1,0,0)$. We next choose $\left(u_{22}, u_{32}\right)=(1,0)$, so that

$$
-q\left(u_{1}, 1,0\right)=\left|a_{11}\right|\left(u_{1}+d_{2}^{\prime}\right)^{2}-\left|a_{11}\right|^{-1}\left(\left|a_{11}\right| A\right)
$$

for appropriate $d_{2}^{\prime}$, where

$$
\begin{aligned}
0<\left|a_{11}\right| A< & \sqrt{ }\left(\frac{5}{4}\left|a_{11}\right|\right), \text { by (19), } \\
& <\frac{5}{8}+\frac{1}{2}\left|a_{11}\right|
\end{aligned}
$$

by the inequality of the arithmetic and geometric means. Since $0<\left|a_{11}\right|<1$, we have

$$
\left|a_{11}\right|\left(2-\left|a_{11}\right|\right)<\frac{3}{2}
$$

i.e.

$$
\frac{5}{8}+\frac{1}{2}\left|a_{11}\right|<1+\frac{1}{4} a_{11}^{2}
$$


and hence

$$
0<\left|a_{11}\right| A<1+\frac{1}{4} a_{11}^{2} .
$$

By Lemma 2, with $a=\left|a_{11}\right|, t^{2}=\left|a_{11}\right| A$, we can always choose an integer $u_{12}$ satisfying

$$
\left|q\left(u_{12}, 1,0\right)\right|<1 \text {. }
$$

Finally, we take $\left(u_{23}, u_{33}\right)=(0,1)$, so that

$$
-q\left(u_{1}, 0,1\right)=\left|a_{11}\right|\left(u_{1}+d_{3}^{\prime}\right)^{2}-\left|a_{11}\right|^{-1}\left(\left|a_{11}\right| C\right)
$$

for some constant $d_{3}^{\prime}$. We now show, with the help of Lemma 5 , that $A$ cannot be too small, and then deduce that $\left|a_{11}\right| C$ is bounded above in terms of $\left|a_{11}\right|$.

Consider the quadratic section

$$
q\left(u_{1}, u_{2}, 0\right)=-\left|a_{11}\right|\left(u_{1}+d_{2}^{\prime} u_{2}\right)^{2}+A u_{2}^{2}
$$

of $q\left(u_{1}, u_{2}, u_{3}\right)$. This is an indefinite quadratic form in $u_{1}, u_{2}$ of determinant $-\left|a_{11}\right| A$, which does not represent zero non-trivially. Thus if $P, N$ denote the lower bounds of the positive values of $q\left(u_{1}, u_{2}, 0\right),-q\left(u_{1}, u_{2}, 0\right)$, respectively, it follows, by Lemma 5 , that

$$
P N \leqq \frac{4}{5}\left|a_{11}\right| A .
$$

By hypothesis,

$$
P \geqq 1 \text { and } N \geqq M>\left|a_{11}\right|(1-\varepsilon) .
$$

Thus by (20), (21) we have

$$
\left|a_{11}\right|(1-\varepsilon)<M \leqq N \leqq P N \leqq \frac{4}{5}\left|a_{11}\right| A,
$$

and hence

$$
A>\frac{5}{4}(1-\varepsilon) .
$$

But since $|2 B| \leqq A \leqq C$, by (19), we have

$$
\frac{3}{4} \cdot \frac{5}{4}(1-\varepsilon) C<\frac{3}{4} A C \leqq A C-B^{2}=\frac{25}{27\left|a_{11}\right|},
$$

which leads to

$$
\left|a_{11}\right| C<\frac{80}{81(1-\varepsilon)}<1+\frac{1}{4} a_{11}^{2},
$$

since $\varepsilon<1 / 81$. A final application of Lemma 5 , with $a=\left|a_{11}\right|$ and $t^{2}=\left|a_{11}\right| C$, shows that

$$
\left|q\left(u_{13}, 0,1\right)\right|<1
$$

for some integer $u_{13}$. The inequalities (17) now follow, with the triads $(1,0,0),\left(u_{12}, 1,0\right)$ and $\left(u_{13}, 0,1\right)$. 
Note. If $M^{\prime}$ denotes the lower bound of the positive values of $q\left(u_{1}, u_{2}, u_{3}\right)$ taken over all integer triads $\left(u_{1}, u_{2}, u_{3}\right) \neq(0,0,0)$, then, by a theorem of Barnes [2], we have

$$
M^{\prime} \leqq\left(\frac{4}{3} \cdot \frac{25}{27}\right)^{1 / 3}=\left(\frac{100}{81}\right)^{1 / 3} \text {. }
$$

It may be remarked that this is inadequate to ensure that $0<a_{11}<1$ and thereby exclude case (ii) of Theorem 2*.

\section{REFERENCES}

1. E. S. Barnes, The minimum of the product of two values of a quadratic form, I, II and III, Proc. London Math. Soc. (3) 1 (1951), 257-283, 385-414, 415-434.

2. E. S. Barnes, The non-negative values of quadratic forms, Proc. London Math. Soc. (3) 5 (1955), 185-196, Theorem 1.

3. J. H. H. Chalk, A theorem of Minkowski on the product of two linear forms, Proc. Cambridge Phil. Soc. 49 (1953), 413-420.

4. J. H. H. Chalk, On the product of $n$ homogeneous linear forms, Proc. London Math. Soc. (3) 5 (1955), 449-473.

5. J. H. H. Chalk, Integral bases for quadratic forms, Canad. J. Math. 15 (1963), 412-421.

6. J. H. H. Chalk and C. A. Rogers, On the product of three homogeneous linear forms, Proc. Cambridge Phil. Soc. 47 (1951), 251-259.

7. H. Davenport, Non-homogeneous ternary quadratic forms, Acta Math. 80 (1948), 65-95; see also Barnes and Swinnerton-Dyer, Inhomogeneous minima of binary quadratic forms (I), Acta Math. 85 (1952), 259-323, especially $\$ 6$.

8. L. E. Dickson, Introduction to the theory of numbers (Chicago, 1929).

9. L. E. Dickson, Studies in the theory of numbers (Chicago, 1930).

10. A. Korkine and G. Zolotareff, Sur les formes quadratiques, Math. Ann. 6 (1873), 366-389; see also [9], Theorem 83.

11. A. M. Macbeath, A new sequence of minima in the geometry of numbers, Proc. Cambridge Phil. Soc. 47 (1951), 266-273.

12. A. Markoff, Sur les formes quadratiques binaires indéfinies, Math. Ann. 15 (1879), 381-406; see also Math. Ann. 56 (1903), 233-251; see also [8], Theorem 119.

13. H. Minkowski, Ueber die Annäherung an eine reele Größe dursch rationale Zahlen, Math. Ann. 54 (1900), 91-124.

14. A. Oppenheim, Values of quadratic forms, I, Quart. J. Math. Oxford Ser. (2) 4 (1953), 54-59, Theorem 1.

15. A. Oppenheim, On indefinite binary quadratic forms, Acta Math. 91 (1954), 43-50.

St. Salvator's College

UNIVERSITY OF ST. ANDREWS 